\title{
Time domain simulation of nonlinear acoustic beams generated by rectangular pistons with application to harmonic imaging
}

\author{
Xinmai Yang and Robin O. Cleveland ${ }^{\text {a) }}$ \\ Department of Aerospace and Mechanical Engineering, Boston University, Boston, Massachusetts 02215
}

(Received 10 June 2004; revised 11 October 2004; accepted 13 October 2004)

\begin{abstract}
A time-domain numerical code (the so-called Texas code) that solves the Khokhlov-ZabolotskayaKuznetsov (KZK) equation has been extended from an axis-symmetric coordinate system to a three-dimensional (3D) Cartesian coordinate system. The code accounts for diffraction (in the parabolic approximation), nonlinearity and absorption and dispersion associated with thermoviscous and relaxation processes. The 3D time domain code was shown to be in agreement with benchmark solutions for circular and rectangular sources, focused and unfocused beams, and linear and nonlinear propagation. The 3D code was used to model the nonlinear propagation of diagnostic ultrasound pulses through tissue. The prediction of the second-harmonic field was sensitive to the choice of frequency-dependent absorption: a frequency squared $f^{2}$ dependence produced a second-harmonic field which peaked closer to the transducer and had a lower amplitude than that computed for an $f^{1.1}$ dependence. In comparing spatial maps of the harmonics we found that the second harmonic had dramatically reduced amplitude in the near field and also lower amplitude side lobes in the focal region than the fundamental. These findings were consistent for both uniform and apodized sources and could be contributing factors in the improved imaging reported with clinical scanners using tissue harmonic imaging. () 2005 Acoustical Society of America.
\end{abstract}

[DOI: $10.1121 / 1.1828671]$

PACS numbers: 43.25.Cb, 43.35.Bf, 43.80.Qf [MFH]

Pages: $113-123$

\section{INTRODUCTION}

High amplitude sound waves are used in many applications. Two examples in biomedical acoustics are focused ultrasound surgery ${ }^{1-3}$ and diagnostic imaging. ${ }^{4,5}$ At the amplitudes used in these applications, effects associated with nonlinear distortion become important, for example, extra heating in tissue ${ }^{6}$ and improved imaging capabilities. ${ }^{7-9} \mathrm{Re}-$ alistic simulations for these applications need to account for three-dimensional (3D) propagation because (1) the sources that are used, particularly in imaging, generate acoustic fields that are 3D and (2) tissue is inhomogeneous ${ }^{10}$ and even for a perfect axis-symmetric source it will result in acoustic fields that are inherently 3D.

A popular model for the propagation of nonlinear directive sound beams is the Khokhlov-Zabolotskaya-Kuznetsov (KZK) equation. ${ }^{11,12}$ This equation accounts for diffraction (in the parabolic approximation), nonlinearity, and thermoviscous absorption. It can be modified to account for arbitrary absorption laws, ${ }^{13}$ sound speed inhomogeneities, ${ }^{14}$ and media with convection. ${ }^{15}$ Numerical solutions of the axissymmetric version (two spatial dimensions) of the KZK equation for a circular source have been widely investigated. ${ }^{13,16-19}$ Numerical solutions for nonlinear axissymmetric beams not restricted to the paraxial region have also been reported. ${ }^{20-23}$ However, calculations for the non axis-symmetric case, for example, the field from a rectangular source, have been limited by the added computational cost of the extra dimension.

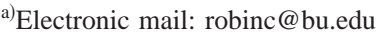

Computer technology has now advanced to the point that realistic three-dimensional problems in diagnostic ultrasound can be solved. Christopher ${ }^{9}$ reported results using a frequency domain code, which accounts for diffraction exactly using the angular spectrum method, to simulate the propagation of finite-amplitude ultrasound through inhomogeneous tissue. Christopher's algorithm has been used by others to consider harmonic leakage in tissue harmonic imaging $^{24}$ and pulse-inversion harmonic imaging. ${ }^{25}$ The socalled Bergen code, a frequency domain solution of the KZK equation, has also been used to simulate the propagation of ultrasonic beams of finite amplitude from rectangular and square sources. ${ }^{26,27}$ Recently a three-dimensional timedomain code has been shown to be in agreement with measurements made in water of the pressure field generated by a clinical scanner. ${ }^{28}$

The main purpose of the present article is to describe a time-domain numerical code capable of simulating the propagation of ultrasonic beams of finite amplitude from a rectangular source. The 3D time-domain code was adapted from an algorithm developed by Lee and Hamilton for axissymmetric sources ${ }^{17}$-the so-called "Texas code." The 3D code accounts for the combined effects of diffraction, nonlinearity, thermoviscous absorption, and multiple relaxation phenomena. ${ }^{29}$ Relaxation processes allow for frequencydependent absorption and dispersion effects to be incorporated into the model. The code was used to consider spatial distribution of the fundamental and second-harmonic signal in tissue for an ultrasound-like imaging scenario and the importance of using the appropriate frequency-dependent absorption is demonstrated. The extension of the code to model 


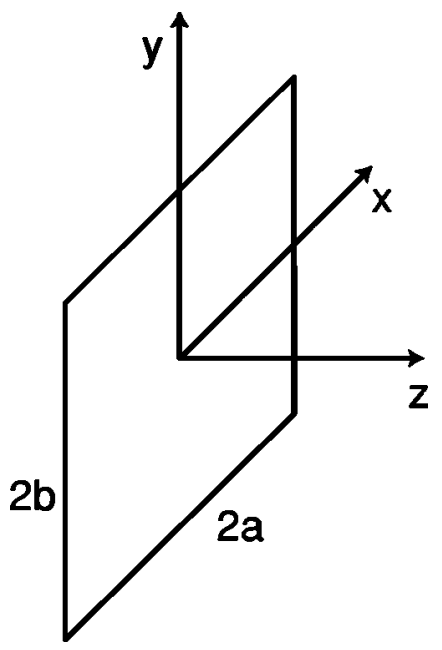

FIG. 1. Geometry and system of coordinates of a rectangular piston.

the propagation of nonlinear beams through an inhomogeneous medium is discussed.

\section{MODEL EQUATION AND NUMERICAL METHOD}

The KZK equation is a model for the paraxial region of a directive nonlinear sound beam. The original equation was developed for thermoviscous fluids, with a frequency squared absorption, but can be extended to fluids with arbitrary absorption and dispersion. ${ }^{13}$ In Cartesian coordinates, the KZK equation, for a relaxing fluid, can be written in the following form:

$$
\begin{aligned}
\frac{\partial p}{\partial z}= & \frac{c_{0}}{2} \int_{-\infty}^{t^{\prime}}\left(\frac{\partial^{2} p}{\partial x^{2}}+\frac{\partial^{2} p}{\partial y^{2}}\right) d t^{\prime \prime}+\frac{\beta}{2 \rho_{0} c_{0}^{3}} \frac{\partial p^{2}}{\partial t^{\prime}}+\frac{\delta}{2 c_{0}^{3}} \frac{\partial^{2} p}{\partial t^{\prime 2}} \\
& +\sum_{v} \frac{c_{v}^{\prime}}{c_{0}^{2}} \int_{-\infty}^{t^{\prime}} \frac{\partial^{2} p}{\partial t^{\prime \prime 2}} e^{-\left(t^{\prime}-t^{\prime \prime}\right) / t_{v}} d t^{\prime \prime},
\end{aligned}
$$

where $p$ is the sound pressure, $z$ the coordinate along the axis of the beam, $x, y$ the transverse coordinates (see Fig. 1), $t^{\prime}=t-z / c_{0}$ the retarded time, $c_{0}$ the small-signal sound speed (in the presence of dispersion this is the equilibrium, $f \rightarrow 0$, sound speed), $\delta$ the diffusivity of sound, $\rho_{0}$ the density, $\beta$ the coefficient of nonlinearity, $t_{v}$ the relaxation time, and $c_{v}^{\prime}$ the small-signal sound speed increment for each of the relaxation processes $v$ (where $v=1,2, \ldots$ ). The terms on the right-hand side account for diffraction, nonlinearity, thermoviscous absorption, and relaxation, respectively.

We obtain a numerical solution to Eq. (1) following the method of Lee and Hamilton. ${ }^{17,29}$ First, Eq. (1) is transformed into nondimensional form on a Cartesian grid; the rationale for the Cartesian grid is discussed in Sec. II B. The following dimensionless variables are introduced:

$\sigma=z / d, \quad X=x / a, \quad Y=y / b, \quad \tau=\omega_{0} t^{\prime}, \quad P=p / p_{0}$,

where $d$ is a characteristic length in the direction of propagation (e.g., the focal length), the quantities $a$ and $b$ are the characteristic lengths in the $x$ and $y$ direction (e.g., the size of the source aperture), $\omega_{0}$ is a characteristic angular frequency (e.g., working frequency of the source pulse), and $p_{0}$ is a characteristic pressure (e.g., peak source pressure). Substitution of Eq. (2) into Eq. (1) yields

$$
\begin{aligned}
\frac{\partial P}{\partial \sigma}= & \frac{1}{4} \int_{-\infty}^{\tau}\left(\frac{1}{G_{x}} \frac{\partial^{2} P}{\partial X^{2}}+\frac{1}{G_{y}} \frac{\partial^{2} P}{\partial Y^{2}}\right) d \tau^{\prime}+N P \frac{\partial P}{\partial \tau}+A \frac{\partial^{2} P}{\partial \tau^{2}} \\
& +\sum_{v} D_{v} \int_{-\infty}^{\tau} \frac{\partial^{2} P}{\partial \tau^{\prime 2}} e^{-\left(\tau-\tau^{\prime}\right) / \theta_{v}} d \tau^{\prime}
\end{aligned}
$$

where the dimensionless coefficients are

$$
\begin{aligned}
& G_{x}=k_{0} a^{2} / 2 d, \quad G_{y}=k_{0} b^{2} / 2 d, \quad N=d / \bar{z}, \\
& A=\alpha_{0} d, \quad D_{v}=k_{0} d c_{v}^{\prime} / c_{0}, \quad \theta_{v}=\omega_{0} t_{v} .
\end{aligned}
$$

Here $k_{0}=\omega_{0} / c_{0}$ is the wave number, $\bar{z}=\rho_{0} c_{0}^{3} / \beta \omega_{0} p_{0}$ is the plane wave shock formation distance, and $\alpha_{0}=\delta \omega_{0}^{2} / 2 c_{0}^{3}$ is the thermoviscous absorption coefficient $(\mathrm{Np} / \mathrm{m})$ at the characteristic frequency.

Equation (3) is solved by marching in the principal propagation direction, $\sigma$. Operator splitting ${ }^{17,30}$ is employed to separate Eq. (3) into the following:

$$
\begin{aligned}
& \frac{\partial P}{\partial \sigma}=\frac{1}{4} \int_{-\infty}^{\tau} \frac{1}{G_{x}} \frac{\partial^{2} P}{\partial X^{2}} d \tau^{\prime}, \\
& \frac{\partial P}{\partial \sigma}=\frac{1}{4} \int_{-\infty}^{\tau} \frac{1}{G_{y}} \frac{\partial^{2} P}{\partial Y^{2}} d \tau^{\prime}, \\
& \frac{\partial P}{\partial \sigma}=N P \frac{\partial P}{\partial \tau}, \\
& \frac{\partial P}{\partial \sigma}=A \frac{\partial^{2} P}{\partial \tau^{2}}, \\
& \left(1+\theta_{v} \frac{\partial}{\partial \tau}\right) \frac{\partial P}{\partial \sigma}=\theta_{v} D_{v} \frac{\partial^{2} P}{\partial \tau^{2}}, \quad v=1,2, \ldots .
\end{aligned}
$$

Each of these equations is solved sequentially at each marching step, $\Delta \sigma$. Equations (4a) and (4b) account for the effect of diffraction, Eq. (4c) the nonlinearity, Eq. (4d) thermoviscous absorption, and Eq. (4e) accounts for the effects of a finite number of individual relaxation processes.

The operator splitting methodology is valid if the step size is small enough that each effect introduces a small correction to the wave form. ${ }^{30}$ For this reason it is numerically advantageous if the characteristic distance $d$ is based on the length scale of the dominant process in the problem. If the step size $\Delta \sigma$ is chosen to be small enough to capture the dominant process, then it will also capture the dynamics of the other processes. For the case of ultrasound propagation in tissue (transducer characteristics: $15 \mathrm{~mm} \times 35 \mathrm{~mm}$ aperture, 3.5 MHz working frequency, and $1 \mathrm{MPa}$ source pressure) the approximate scales are absorption length $60 \mathrm{~mm}$, shock formation distance $30 \mathrm{~mm}$, focal length $70 \mathrm{~mm}$, and Rayleigh distance $1200 \mathrm{~mm}$. We note that the first three length scales have similar values, which means that any of them is an appropriate candidate for the characteristic distance $d$. For modeling an ultrasound scanner a convenient characteristic distance is the focal length associated with the elevation plane of the transducer because it is normally a fixed value while the other length scales may vary with either the operating frequency of the transducer or the beam forming. 
In this work two finite-difference schemes were compared for calculating the diffraction effects, Eqs. (4a) and (4b). The first scheme is the same as that used in the twodimensional (2D) Texas code and consisted of the implicit backward finite difference (IBFD) method for the first 100 marching steps $(\Delta \sigma=0.001)$, in order to damp numerical oscillations, and the Crank-Nicolson finite difference (CNFD) method thereafter $(\Delta \sigma=0.01) .{ }^{17,30}$ An extra check is carried out at each step to ensure that the nonlinear distortion will not allow the wave form to become multivalued. ${ }^{17}$ In the $3 \mathrm{D}$ code this is implemented by solving Eqs. (4a) and (4b) independently over each incremental step $\Delta \sigma$; as it is not possible to solve both diffraction terms simultaneously and still retain a system of equations that can be expressed as a tri-diagonal matrix, which has significant advantages from a computational point of view. ${ }^{30}$

The second scheme replaces the CNFD method with the Alternating Direction Implicit (ADI) method. ${ }^{30}$ In the ADI method each diffraction step is broken into two half-steps: at the first half-step one transverse coordinate is solved implicitly and the other explicitly and this is alternated at the second half-step. In this case it is possible to combine Eqs. (4a) and $(4 b)$ to

$$
\frac{\partial P}{\partial \sigma}=\frac{1}{4} \int_{-\infty}^{\tau}\left(\frac{1}{G_{x}} \frac{\partial^{2} P}{\partial X^{2}}+\frac{1}{G_{y}} \frac{\partial^{2} P}{\partial Y^{2}}\right) d \tau^{\prime},
$$

and still produce a tri-diagonal system at each half-step. This means for both CNFD and ADI it is necessary to solve two diffusion-like equations at each full step. The full step size of the ADI is the same as the CNFD and we found it was still necessary to employ the IBFD method for the first 100 marching steps in order to damp numerical oscillations. For both schemes the other equations are solved in the manner developed in the axis-symmetric Texas code. ${ }^{17,29}$ Briefly, Eq. (4c) is solved exactly using the Poisson solution. Equations (4d) and (4e) are solved using initially an IBFD method followed, after the same 100 step transition, by the CNFD method.

\section{A. Source conditions}

The source condition for the KZK equation is determined by defining the time wave forms at $\sigma=0$ for all $X$ and $Y$. In general the code is capable of having arbitrary excitation applied at the source location. Three source conditions were considered in this study.

(1) An unfocused uniformly excited piston, where the source condition (in dimensional variables) was expressed in the following form:

$$
p=p_{0} f(t) g(x, y) \quad \text { at } z=0 \text {, }
$$

where $f(t)$ was the source wave form and $g(x, y)$ the amplitude shading function. For uniform shading the function $g$ is the two-dimensional top hat function, which in dimensionless co-ordinates is

$$
g(X, Y)=\left\{\begin{array}{l}
1,-1 \leqslant X \leqslant 1 \text { and }-1 \leqslant Y \leqslant 1 \\
0, \text { otherwise. }
\end{array}\right.
$$

(2) A focused sound beam, where the source condition can be written within the parabolic approximation as follows:

$$
p=p_{0} f\left(t+x^{2} / 2 c_{0} d_{X}+y^{2} / 2 c_{0} d_{Y}\right) g(x, y) \quad \text { at } z=0 .
$$

In dimensionless variables this source condition takes the form:

$$
P=f\left(\tau+G_{X S} X^{2}+G_{Y S} Y^{2}\right) g(X, Y) \quad \text { at } \sigma=0,
$$

where $G_{X S}=G_{X} d / d_{X}$ and $G_{Y S}=G_{Y} d / d_{Y}$ ensure the correct phase in the source wave form to effect focusing at distance $d_{X}$ in the $x z$ plane and $d_{Y}$ in the $y z$ plane. The shading function $g(X, Y)$ was taken as a top-hat function.

(3) An apodized focused sound beam, where the focusing was effected using Eq. (8) but the amplitude was shaded using a cosine function in the $X$ direction and thus impacting primarily the scan plane. The shading function used was

$$
\begin{aligned}
& g(X, Y)=g_{1}(X) g_{2}(Y), \\
& g_{1}(X)= \begin{cases}\cos \left(\frac{\pi}{2} X\right), \quad-1 \leqslant X \leqslant 1 \\
0, & \text { otherwise }\end{cases} \\
& g_{2}(Y)= \begin{cases}1, & -1 \leqslant Y \leqslant 1 \\
0, & \text { otherwise. }\end{cases}
\end{aligned}
$$

Apodization is commonly used in imaging systems to reduce side lobe levels.

\section{B. Boundary conditions}

In the lateral directions the Cartesian grid extended from $X_{\min }$ to $X_{\max }$ in the $x$ axis with uniform spacing $\Delta X$ and from $Y_{\min }$ to $Y_{\max }$ in the $y$ axis with uniform spacing $\Delta Y$. A zero pressure boundary condition was applied along the edges of the numerical domains, that is, at $X=X_{\max }, X=X_{\min }, Y$ $=Y_{\max }$, and $Y=Y_{\min }$. The zero pressure boundary condition was simple to implement but did result in reflections from the edge of the numerical grid that can interfere with the desired signals in the central part of the grid. The time domain code described here used pulses and in this work the numerical boundaries were placed far enough away that the reflections did not interfere with the signals of interest. This is analogous to an experimental system where physical boundaries need to be far enough away to prevent real echoes affecting measurements. The trade-off with this approach is that the outer boundaries may need to be placed at very large distances to ensure that reflections do not interfere with the field of interest, which can result in a large computational cost particularly for long tone bursts.

One method of reducing the size of the grid and yet still avoid reflections is to use a transformed spatial grid whereby the outer boundary is allowed to move as the sound beam either focuses or spreads. A reflection is still generated but the boundary is placed such that the amplitude of the reflection is small. For example, in the case of an unfocused piston a spreading grid that approximates the field of a circular source in the linear limit has been used ${ }^{31}$ and for highly focused beams a mixed converging/diverging grid has been used. $^{32,33}$ In this work we anticipate applying the algorithm 
to the problem of biomedical ultrasound imaging which is a low focusing problem (typical gains around 3 to 10) and transforming the equation does not provide a significant advantage. ${ }^{34}$ Therefore, the KZK equation was solved directly on a Cartesian grid without transformation.

We note that an alternative method to reduce boundary reflections is to employ an absorbing boundary layer at the outer edge of the grid. In this case the outer edge of the domain is approximated to appear as an infinite space. Examples include using the plane wave impedance condition, ${ }^{35}$ application of a tapered spatial window, ${ }^{26,36}$ or a perfectly matched layer which can be designed to absorb the sound before reflections occur. ${ }^{37}$ Absorbing boundary layers allow the size of the numerical grid to be reduced but they add a level of complexity to the code. For the simulations investigated here, which involved short time pulses, it was possible to obtain solutions in a timely manner without implementing an absorbing boundary layer.

In special cases where it is known a priori that the field has symmetry, internal boundaries can be introduced to reduce the size of the numerical domain. For the case of symmetric sources, e.g., square or rectangular, propagating into a homogeneous or layered medium, it is possible to halve the size of the numerical domain for each axis of symmetry by placing an artificial rigid boundary along the axis. Therefore, in the case of a square or rectangular piston it is necessary to solve for only one quadrant of the $X-Y$ space. At each axis of symmetry one of the following boundary conditions was applied: $\partial P /\left.\partial X\right|_{X=0}=0$ or $\partial P /\left.\partial Y\right|_{Y=0}=0$. In the numerical implementation the second-order derivative at boundary was determined from a Taylor series expansion around the axis:

$$
\begin{aligned}
P(\Delta X)= & \left.p\right|_{X=0}+\Delta X\left(\frac{\partial P}{\partial X}\right)_{X=0}+\frac{(\Delta X)^{2}}{2}\left(\frac{\partial^{2} P}{\partial X^{2}}\right)_{X=0} \\
& +O\left((\Delta X)^{3}\right) .
\end{aligned}
$$

Setting the first-order derivative to zero yields

$$
\left.\frac{\partial^{2} P}{\partial X^{2}}\right|_{X=0}=2 \frac{P(\Delta X)-P(0)}{(\Delta X)^{2}}+O(\Delta X) .
$$

We note that this approximation is correct only to first-order in space, whereas all other finite-difference approximations are correct to second-order in space. Increasing the accuracy of this expression to second-order would result in losing the tri-diagonal nature of the algorithm, so the first-order expression was used in our simulations. An identical trade-off is made in the axis-symmetric Texas code. The 3D code was written to be able to include either axis of symmetry as required.

\section{NUMERICAL RESULTS}

The results from the code were benchmarked to other solutions in the literature for both circular pistons and rectangular pistons. The code was then used to simulate the propagation of a diagnostic ultrasound beam in tissue.
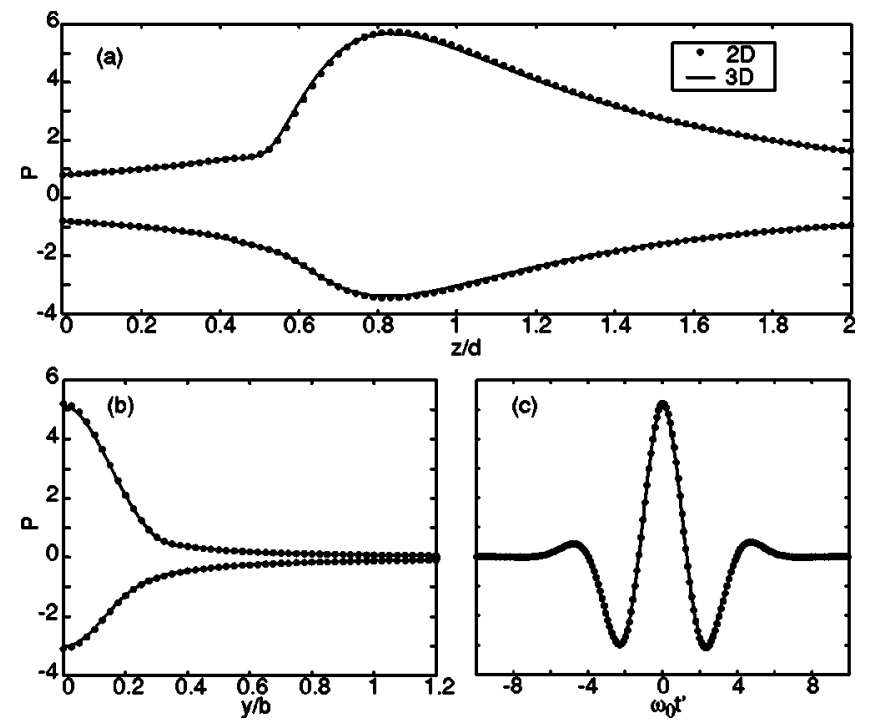

FIG. 2. Linear propagation from a circular piston: $2 \mathrm{D}$ vs $3 \mathrm{D}$. (a) axial amplitude ( $z$ axis), (b) transverse beam plots at $\sigma=1 / 0$, (c) wave forms at $\sigma=1.0$.

\section{A. Focused circular source: Linear and nonlinear}

First, results of the 3D code were compared with the 2D Texas code for a focused circular symmetric source of radius $a$ and focal length $d$. The source wave form was a smooth pulse given by ${ }^{17}$

$$
P=\exp \left[-(\tau / n \pi)^{2 m}\right] \sin \tau \text {. }
$$

In this section the number of cycles $n=0.9549$ and the envelope exponent $m=1$ resulted in a short pulse about onecycle in duration. The focusing gain of the transducer was taken to be $G=5.0$. The time window stretched from $\tau_{\text {min }}$ $=-20 \pi$ to $\tau_{\max }=20 \pi$ with a sampling rate $\Delta \tau=2 \pi / 60$, that is, there were 60 points per cycle and 1200 points in the time window. In the $2 \mathrm{D}$ code the grid had a maximum radius $\rho_{\max }=r / a=4$ and there were 160 grid points in radial direction $(\Delta \rho=0.025)$. In the 3D simulation the symmetry of the problem allowed us to solve the problem on a quadrant with a lateral grid given by $0 \leqslant X \leqslant X_{\max }$ or $0 \leqslant Y \leqslant Y_{\max }$ with $X_{\max }=Y_{\max }=4$ and 160 grid points in the $X$ and $Y$ directions $(\Delta X=\Delta Y=0.025)$. The step size was $\Delta \sigma=0.001$ for IBFD and $\Delta \sigma=0.01$ for CNFD. The memory requirement of the axis-symmetric code was on the order of 800000 unknowns per marching step, for the $3 \mathrm{D}$ code the requirement was 500000000 unknowns per marching step. The memory and computational requirements became almost 1000 times larger by solving this particular problem in 3D.

The first scenario considered a linear lossless problem $(A=0, N=0)$. Figure 2 compares axial propagation curves, focal beam patterns, and focal wave forms from the two codes. There is excellent agreement between the results of the 2D and 3D codes. A slight discrepancy exists in the positive peak of the wave form in Fig. 2(c). The discrepancy was the result of the extra dimension of discretization used in the 3D code (recall the 2D code assumed cylindrical symmetry and introduced no discretization error in the $\theta$ coordinate) and we found it could be reduced by using more lateral grid points in the 3D code. 

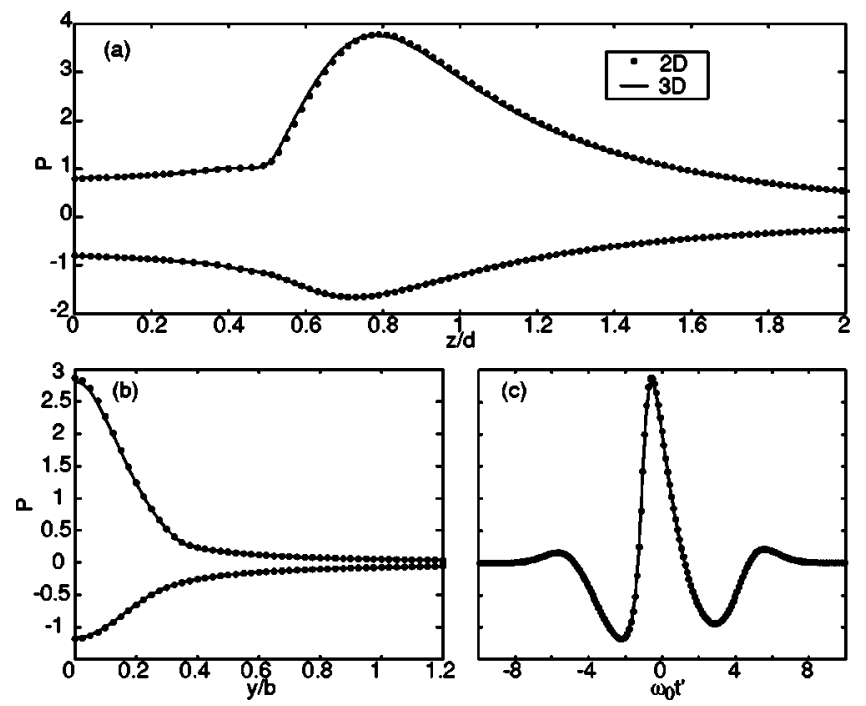

FIG. 3. Nonlinear propagation from a circular piston: 2D vs 3D. (a) Axial amplitude ( $z$ axis), (b) transverse beam plots at $\sigma=1 / 0$, (c) wave forms at $\sigma=1.0$.

Figure 3 shows a similar comparison for the same source condition and grid but for a case with nonlinearity and absorption present. The dimensionless nonlinearity and absorption coefficients were $N=1.0$ and $A=0.5$. The agreement is again excellent except in Fig. 3(c) where the 3D code slightly underpredicted the peak positive pressure due to the extra discretization in the $3 \mathrm{D}$ code.

\section{B. Unfocused rectangular source: Linear and nonlinear}

For the case of a uniform unfocused rectangular piston, we compared the result of a linear, lossless calculation using the $3 \mathrm{D}$ code with the results of the Rayleigh integral. We used the solution and excitation pulse of Ullate and San Emeterio. ${ }^{38}$ The excitation was a short pulse of approximately 1.5 cycles at $3 \mathrm{MHz}$ (see Fig. 4) which propagated in water $\left(c_{0}=1500 \mathrm{~m} / \mathrm{s}\right)$. The aperture size was $24 \times 15 \mathrm{~mm}$ ( $a=12 \mathrm{~mm}$ and $b=7.5 \mathrm{~mm}$ ) with a resulting Rayleigh dis-

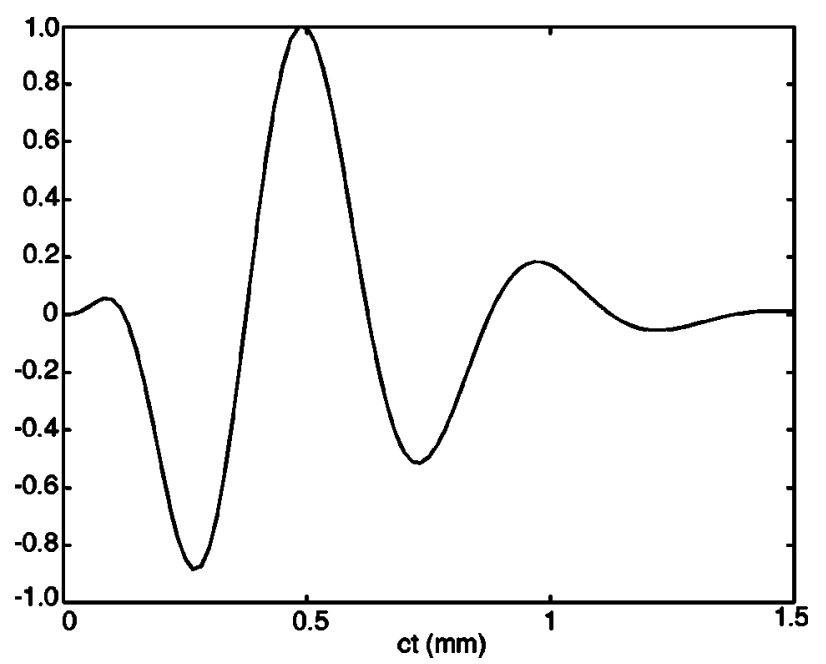

FIG. 4. Source velocity of rectangular piston for comparison with a Rayleigh integral solution (Ref. 38). The central frequency is $f_{0}=3 \mathrm{MHz}$.

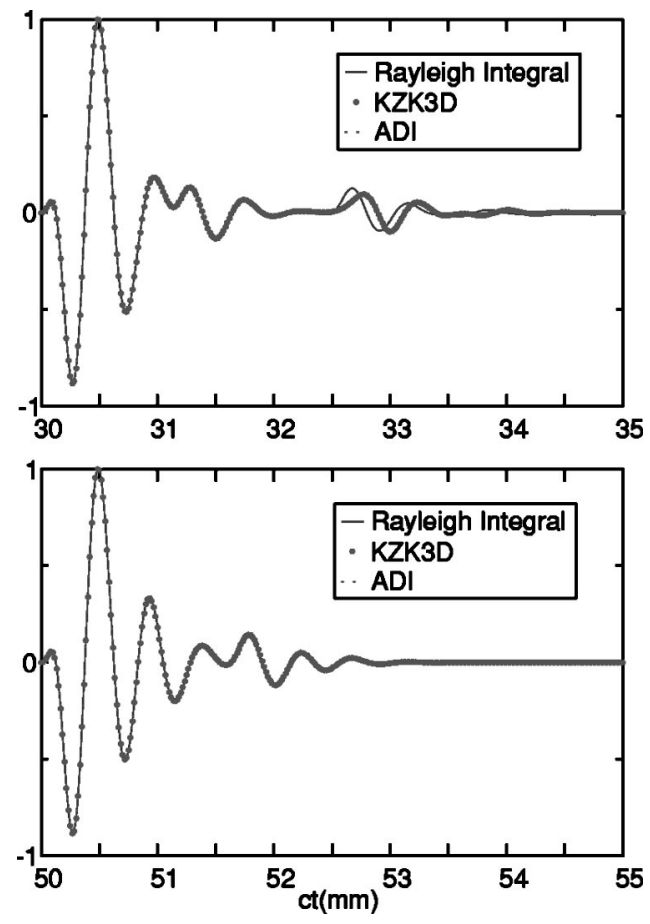

FIG. 5. Predicted axial wave forms at $z=30 \mathrm{~mm}$ (upper) and $z=50 \mathrm{~mm}$ (lower) for linear propagation from a rectangular aperture-comparison of the Rayleigh integral, KZK3D (CNFD) and KZK3D (ADI). The KZK code agrees well with the analytical solution except for the edge wave at $z$ $=30 \mathrm{~mm}$ where the parabolic approximation should start to break-down. There was no significant difference between the CNFD and ADI methods.

tance of $720 \mathrm{~mm}$ which, in the absence of a focal length, was used as the characteristic distance. Neither absorption nor nonlinearity was calculated in the simulations. The time window was from $\tau_{\min }=-20 \pi$ to $\tau_{\max }=20 \pi$ with 30 points per cycle, that is, $\Delta \tau=\pi / 15$. The outer edges of the numerical grid were at $X_{\max }=Y_{\max }=4$ and there were 800 grid points in both the $x$ and $y$ directions $(\Delta X=\Delta Y=0.005)$. The step size was $\Delta \sigma=5 \times 10^{-5}$ for IBFD and $\Delta \sigma=5 \times 10^{-4}$ for CNFD.

Figure 5 compares on-axis wave forms at $z=30 \mathrm{~mm}$ and $z=50 \mathrm{~mm}$ with the Rayleigh integral results. At $z=50 \mathrm{~mm}$ there is excellent agreement between the exact solution and the KZK code. At $z=30 \mathrm{~mm}$, which is well within the near field of this transducer, there is excellent agreement for the portion of the wave that can be identified as arriving from the center of the transducer, but a small error in the arrival time of the edge wave. This discrepancy was due to the use of the parabolic approximation that is incorporated into the $\mathrm{KZK}$ equation. At a range of $30 \mathrm{~mm}$ the half-aperture angle to the corner of the source was $25^{\circ}$ which is beyond what is usually acceptable for the parabolic approximation to be valid and, although rectangular apertures are less sensitive to errors in the edge wave, ${ }^{26}$ the error manifests itself in the arrival time of the edge wave.

In Fig. 5 we also show results obtained using the ADI method on the same grid. The curves completely overlap with the results from IBFD/CNFD. However, the ADI method required about $10 \%$ more CPU time than the IBFD/ CNFD, and nearly twice as much computational memory. Therefore we chose to use IBFD/CNFD algorithms for our code. 

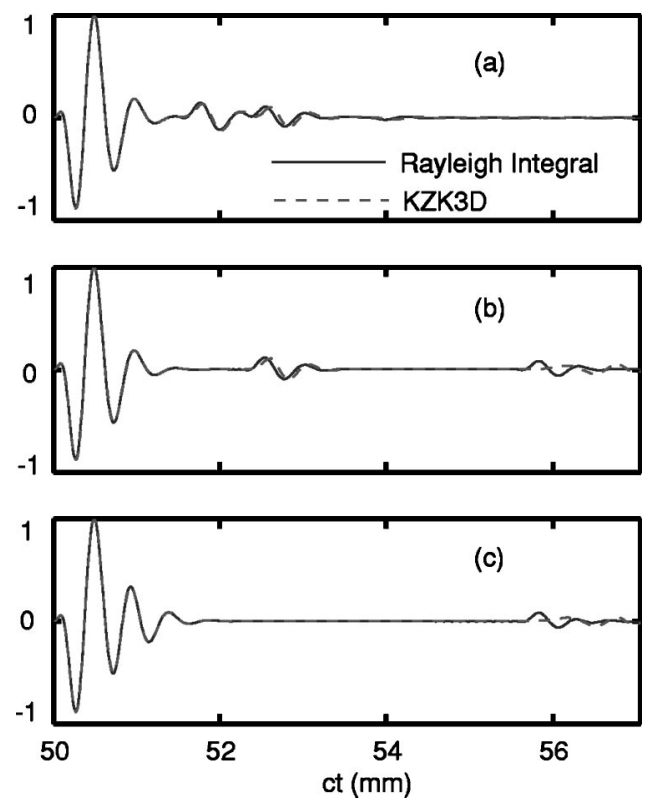

FIG. 6. Comparison of off-axis wave forms from a rectangular aperture at $z=50 \mathrm{~mm}$ for (a) $x=0, y=b$, (b) $x=a, y=b$, and (c) $x=a, y=0$. There is generally excellent agreement between the analytical solution and the KZK3D code-the small discrepancies in the edge wave are attributable to the parabolic approximation.

Figure 6 shows three off-axis wave forms $(x=0, y$ $=b ; x=a, y=b$ and $x=a, y=0)$ at a distance of $z$ $=50 \mathrm{~mm}$. The agreement is generally excellent between the analytical solution and the KZK code with the exception of slight discrepancies in the arrival of the final edge wave. This is because even though at $z=50 \mathrm{~mm}$ the parabolic approximation was fine on axis, the propagation of sound from the furthest points of the source occurred at too great an angle for the parabolic approximation to hold for field points that were substantially off axis.

For the nonlinear pressure field of rectangular apertures, we compared our code with results from the Bergen code for a square aperture given by Baker et al. ${ }^{26}$ The parameters used in the simulation were $c_{0}=1486 \mathrm{~m} / \mathrm{s}, \quad \rho_{0}$ $=1000 \mathrm{~kg} / \mathrm{m}^{3}, \beta=3.5$, fundamental frequency $2.25 \mathrm{MHz}$, and $\alpha_{0} d=0.094$. The time window was from $\tau_{\min }=-20 \pi$ to $\tau_{\max }=20 \pi$ with $\Delta \tau=\pi / 32$ (64 points per cycle), the extent of the lateral grid was $X_{\max }=Y_{\max }=4$ with grid spacing $\Delta X$ $=\Delta Y=0.025$ (160 points in each direction) and $\Delta \sigma=10^{-3}$ for IBFD and $\Delta \sigma=10^{-2}$ for CNFD. The time-domain code was used to simulate $\mathrm{cw}$ performance by using a 10 cycle long pulse [Eq. (10) with $n=10$ and $m=11]$. The amplitude of the harmonics in the time-domain code was determined by calculating the discrete Fourier transform of the center cycle in the pulse. Figure 7 shows the amplitude of the fundamental, second harmonic and third harmonic along the axis for a square aperture with amplitude of $220 \mathrm{kPa}$. We found excellent agreement in the predicted amplitude of the harmonics by the KZK code and the Bergen code.

\section{Focused rectangular source: Nonlinear propagation in tissue}

In this section results are presented for the propagation of a nonlinear ultrasound beam through tissue. In particular

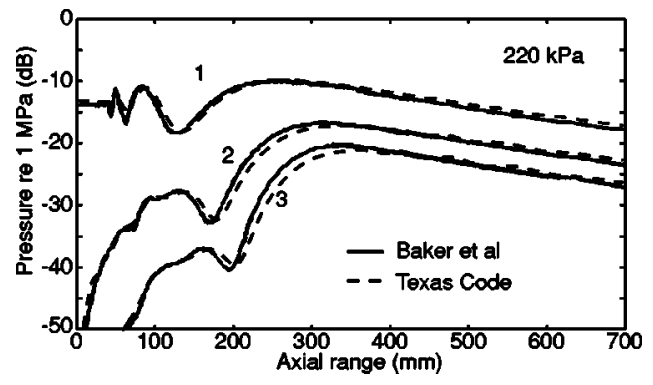

FIG. 7. Amplitude of the fundamental, second, and third harmonic as a function of propagation distance from a rectangular aperture. The results from this code compare favorably with those of Baker et al. (Ref. 26) for the same parameters.

we wish to investigate the spatial distribution of the fundamental and second-harmonic signals in tissue for application to diagnostic imaging. Tissue harmonic imaging (THI) is currently implemented on most mid-range and high-end commercial scanners, and in this mode images are formed from the reflected signals at the second harmonic rather than the fundamental. THI is currently the imaging mode of choice in cardiology where it has been shown to improve border delineation, ${ }^{39,40}$ and measurements of heart function. ${ }^{41}$ THI also appears to be promising in imaging other organs in the body, for example, it offers improved detection of cysts in the breast ${ }^{42}$ and lesions in the liver ${ }^{43}$ and kidney. $^{44}$

THI is reported to provide enhancement in the spatial resolution of targets ("detail contrast") 45 which has been attributed to the smaller focal spot, ${ }^{24,39}$ immunity to aberration effects generated by near field inhomogeneities, 9,24 and nonlinear interaction at interfaces. ${ }^{46} \mathrm{THI}$ is also reported to provide superior "contrast resolution" as it is less sensitive to clutter. $^{24,39}$ Clutter refers to received echo signals that are generated outside the region of interest, for example, reverberation in the near field due to the presence of a fat layer near the skin or scattering by objects in the side-lobes. We investigated possible reasons for the image enhancement based on the distribution of the spatial distribution of the fundamental and second harmonic. We also evaluated the effect of source apodization and different tissue absorption laws on the predictions.

The source was modeled as a rectangular source with dimensions of $15 \mathrm{~mm}$ in the scan plane ( $x$ axis) and $10 \mathrm{~mm}$ in the elevation plane ( $y$ axis). We modeled the source as being continuously phased, that is, no individual array elements, however, the code is capable of modeling arbitrary conditions. The source was given two different geometrical focal distances: $d_{x}=80 \mathrm{~mm}$ in the scan plane (normally done by electronic focusing in an imaging system) and $d_{y}$ $=50 \mathrm{~mm}$ in the elevation plane (normally a fixed focus in an imaging system) as most imaging is done with different focal lengths in the scan and elevation planes. The source was uniformly excited with a short pulse [Eq. (11) with $n=3$ cycles and $m=4]$ and a center working frequency of $2 \mathrm{MHz}$. This wave form is similar to what will be used by a clinical scanner in THI mode, which uses more cycles than would be used in regular B-scan to improve the spectral purity in the source wave form. The pressure at the source was modeled 


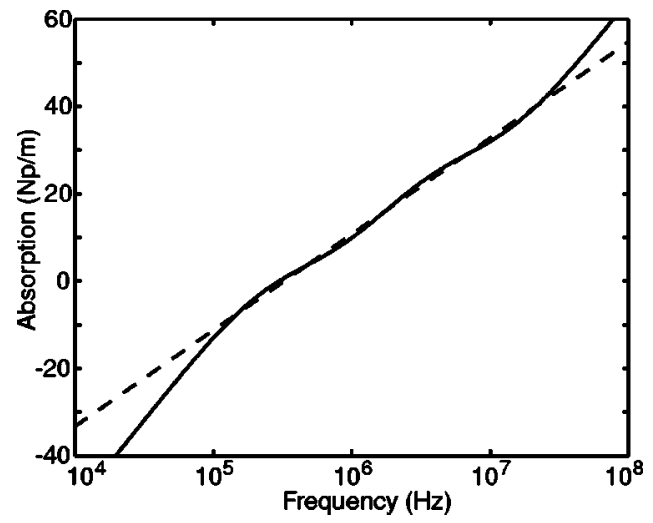

FIG. 8. Comparison of absorption curves for: two-relaxation process fit to a $f^{1.1}$ law (solid line) and thermoviscous absorption $f^{2}$ (dashed line).

to have an amplitude of $0.5 \mathrm{MPa}$ resulting in a mechanical index (MI) in the tissue of approximately 0.7 (note this was based on the peak negative pressure predicted by the simulation and not from derating the peak pressure obtained from a simulation through water).

The tissue was modeled as a homogeneous medium with density $\rho_{0}=1050 \mathrm{~kg} / \mathrm{m}^{3}$ and sound speed $c_{0}=1540 \mathrm{~m} / \mathrm{s}$, and coefficient of nonlinearity $\beta=5$. The absorption was assumed to obey the following power law:

$$
\alpha=\alpha_{0}\left(\frac{f}{f_{0}}\right)^{\eta},
$$

where $\alpha_{0}=3.4538 \mathrm{~Np} / \mathrm{m}, \eta=1.1$, and $f_{0}=1 \mathrm{MHz}$ (equivalent to $0.3 \mathrm{~dB} / \mathrm{cm}$ at $1 \mathrm{MHz}$ ). The time-domain code cannot model such frequency-dependent absorption exactly; however, this behavior can be approximated by using a number of relaxation processes. In this simulation we used two relaxation processes to model the absorption properties of the tissue, that is, the effective absorption coefficient was

$$
\alpha(f)=\frac{c_{R 1}^{\prime}(1-j f)}{f^{2}+f_{R 1}^{2}}+\frac{c_{R 2}^{\prime}(1-j f)}{f^{2}+f_{R 2}^{2}}+\alpha_{\mathrm{TV}} f^{2} .
$$

The parameters $c_{R 1}^{\prime}, f_{R 1}, c_{R 2}^{\prime}, f_{R 2}$, and $\alpha_{T V}$ were chosen to give a best fit, in a least squares sense, to the absorption law given in Eq. (12) over the frequency band of $100 \mathrm{kHz}$ to 30 MHz. The fminsearch function from MATLAB (The Mathworks, Natick, MA) was used to determine the parameters for the two relaxation processes and the thermoviscous absorption coefficient. The fitted parameters were: relaxation frequencies $f_{R 1}=237.0 \mathrm{kHz}$ and $f_{R 2}=3.749 \mathrm{MHz}$; relaxation dispersion $\left(c^{\prime} / c 0\right)_{R 1}=1.3990 \times 10^{-3}$ and $\left(c^{\prime} / c 0\right)_{R 2}$ $=1.6597 \times 10^{-3}$, and the thermoviscous absorption coefficient $\alpha_{\mathrm{TV}}=1.625 \times 10^{-13} \mathrm{~Np} / \mathrm{m} / \mathrm{Hz}^{2}$. The comparison of the power-law absorption and the fit using relaxation processes is shown in Fig. 8. There is excellent agreement over the frequency band of interest. We note that the relaxation operator also correctly accounts for dispersion, that is, the Kramers-Kronig relations are satisfied by the absorption given in expression in Eq. (13). ${ }^{47}$

The dimensionless parameters for this simulation were $N=0.6554, \quad G_{X}=2.869, \quad G_{Y}=1.275, \quad A=0.0520, \quad D_{1}$ $=0.9133, \theta_{1}=8.4377, D_{2}=1.0835$, and $\theta_{2}=0.5334$. The focusing at the source was $G_{X S}=2.869$ and $G_{Y S}=2.04$. The
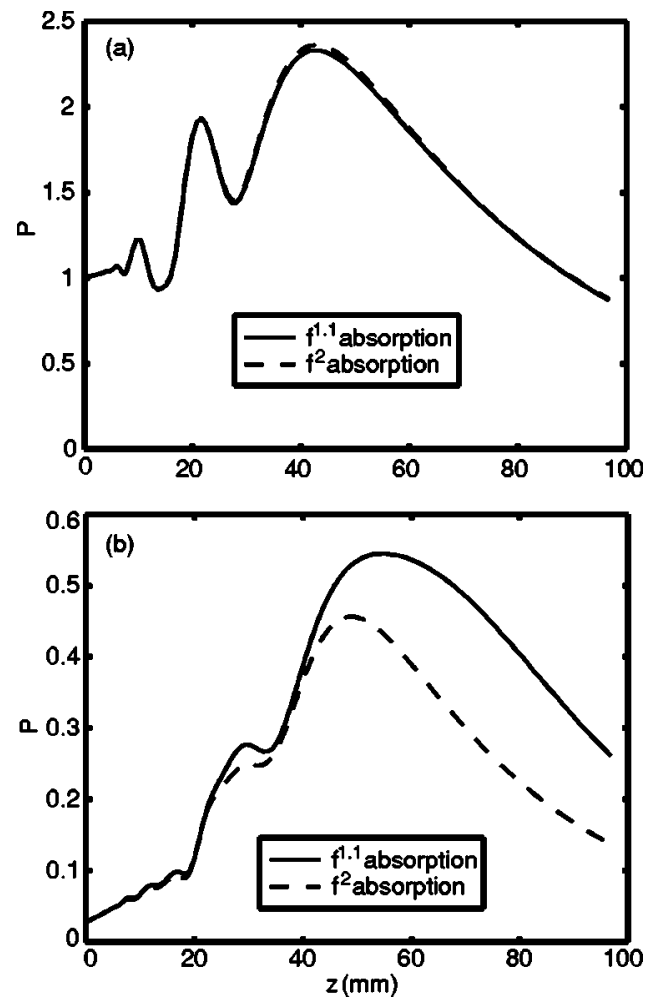

FIG. 9. Simulation of nonlinear propagation in a tissue-like medium. Axial plots of the amplitude of (a) the fundamental and (b) the second harmonic. The solid line is the result for $f^{1.1}$ absorption and the dashed line for $f^{2}$ absorption.

time window was from $\tau_{\min }=-20 \pi$ to $\tau_{\max }=20 \pi$ with $\Delta \tau$ $=2 \pi / 64, X_{\max }=Y_{\max }=4$, and $\Delta X=\Delta Y=0.01$. The step size was $\Delta \sigma=10^{-5}$ for IBFD and $\Delta \sigma=10^{-2}$ for CNFD.

In Fig. 9 we present plots of the axial amplitude of the fundamental and second harmonic in the tissue. In this case the amplitudes were determined by carrying out a discrete Fourier transform on a five-cycle window around the pulse. The solid lines are the predictions for $f^{1.1}$ absorption and the dashed lines for $f^{2}$ absorption. The coefficient of the $f^{2}$ absorption law was chosen to yield the same absorption as the $f^{1.1}$ law at the fundamental frequency of $2 \mathrm{MHz}$. The results show that the absorption law had little impact on the prediction of the fundamental. This is not unexpected as the two absorption laws match at this frequency. However, there was a substantial difference in the prediction of the second harmonic, in particular the peak amplitude of the second harmonic for the $f^{2}$ simulation was reduced by more than $20 \%$ and the location of the peak shifted toward the source by 6 $\mathrm{mm}$. Both of these phenomena are consistent with the fact that $f^{2}$ absorption will attenuate the second harmonic more strongly than $f^{1.1}$ absorption. We note that the amplitude of the fundamental in the $f^{1.1}$ case was slightly lower in amplitude in the focal region which is because the second harmonic has higher amplitude for the $f^{1.1}$ case and this resulted in more efficient transfer of energy from the fundamental to the third and higher harmonics.

Figure 10 shows contour plots of the amplitude of the fundamental and second harmonic of the pulse in the scan plane. The solid lines are the predictions for $f^{1.1}$ absorption and the dashed lines for $f^{2}$ absorption. We see that the off- 

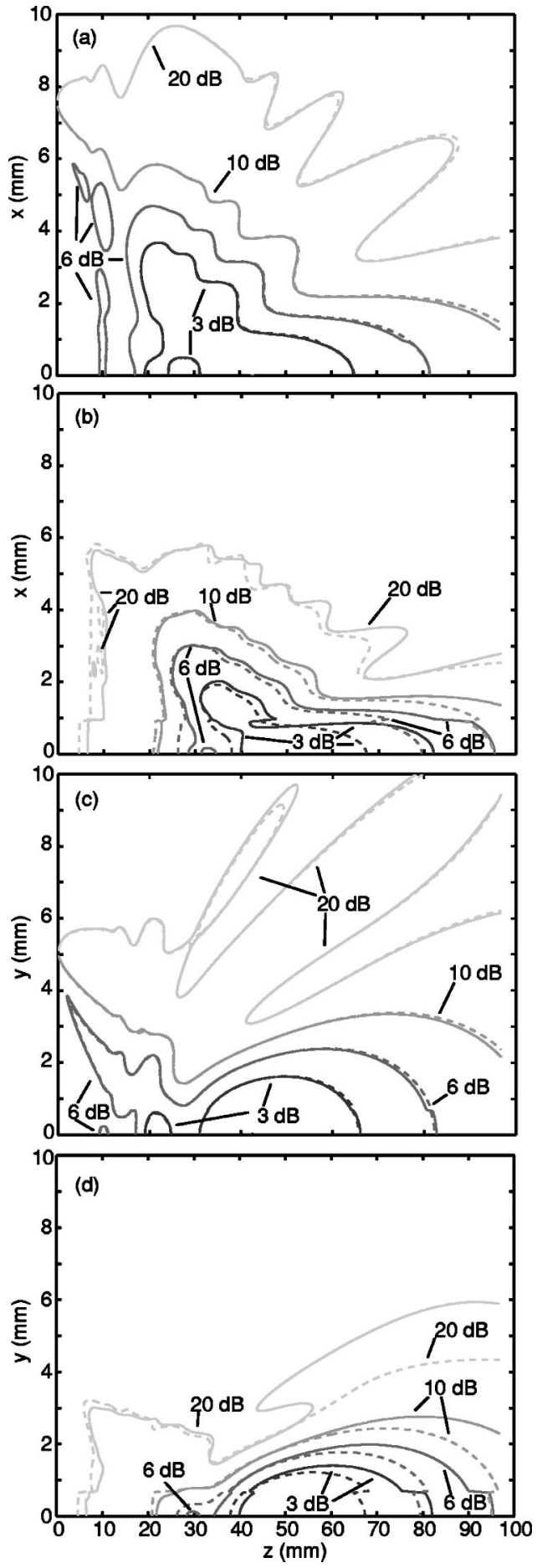

FIG. 10. Simulation of nonlinear propagation in a tissue-like medium. Contour plots of the amplitude of (a) the fundamental in the scan $(x-z)$ plane, (b) the second harmonic in the scan plane, (c) the fundamental in the elevation $(y-z)$ plane, and (d) the second harmonic in the elevation plane. The reference value was the peak amplitude for each condition. The solid line is the result for $f^{1.1}$ absorption and the dashed line for $f^{2}$ absorption.

axis distribution of the second harmonic was not as sensitive as the on-axis distribution to the use of an incorrect absorption. In comparing Figs. 10(a) and 10(b), we note that the second-harmonic signal in the scan plane was more spatially confined than the fundamental. The distance to the $-3 \mathrm{~dB}$ contour was $\sim 0.9 \mathrm{~mm}$ for the second harmonic and $\sim 1.3$ $\mathrm{mm}$ for the fundamental which could contribute to improve-
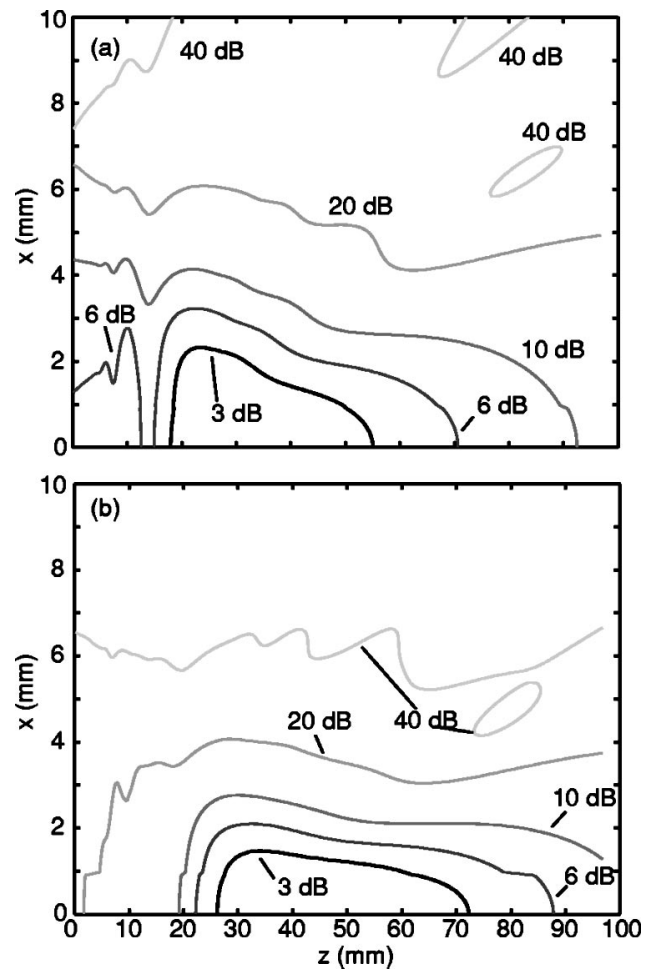

FIG. 11. Simulation of nonlinear propagation in a tissue-like medium $\left(f^{1.1}\right.$ absorption) with a cosine amplitude shading of the source in the scan plane. Contour plots of (a) the fundamental and (b) the second harmonic in the scan $(x-z)$ plane.

ments in detail resolution on-axis. Further, the $-20 \mathrm{~dB}$ contour of the second harmonic barely extends more than $5 \mathrm{~mm}$ from the axis where as for the fundamental it extends up to $10 \mathrm{~mm}$ away which means that the second harmonic should suffer less from clutter signals due to off-axis scatterers. Lastly, in the near field of the transducer the levels of the second harmonic $(\sim-10 \mathrm{~dB}$ at $z=20 \mathrm{~mm})$ are much less than those of the fundamental $(\sim-3 \mathrm{~dB}$ at $z=20 \mathrm{~mm})$ and therefore the second-harmonic signal should suffer from less clutter in the presence of reverberating layers. Figures 10(c) and $10(\mathrm{~d})$ show the contour plots in the elevation plane, and similar features can be observed in these plots. We note that repeating these simulations with a larger grid $X_{\max }=Y_{\max }$ $=6$ produced no changes to the results, and this indicates that the data were not affected by reflections from the edge of the numerical grid.

Finally, we demonstrate the impact of apodization on the field of the fundamental and second harmonic for propagation through tissue (with $f^{1.1}$ absorption law). The source condition described by Eq. (9) was employed. Figure 11 shows contour plots of the fundamental and the second harmonic in the scan plane. Compared to Fig. 10, one observes that the apodization greatly reduced the amplitude of the side lodes of the fundamental as expected. The apodization reduced the "side-lobes" of the second-harmonic signal as well. Contour levels are shown down to $-40 \mathrm{~dB}$ and for the second-harmonic signal the $-40 \mathrm{~dB}$ contour rarely extends to approximately $6 \mathrm{~mm}$ from the axis whereas for the fundamental the $-20 \mathrm{~dB}$ contour lies around $6 \mathrm{~mm}$ off axis and the $-40 \mathrm{~dB}$ contours extends mostly beyond the range 
shown. The effect of the source apodization on the acoustic field in the elevation plane was much less dramatic (data not shown), which is consistent with the fact that no apodization was implemented in that axis. Therefore, even though the beam pattern of the fundamental signal is improved by apodization, the second harmonic still has a narrower beam (with lower side lobes) and much less near field structure than the fundamental.

\section{CONCLUSIONS AND DISCUSSION}

A time-domain algorithm that solves the KZK equation in three spatial dimensions has been described. The code includes the effects of diffraction, absorption, nonlinearity, and relaxation on the propagation of the finite-amplitude sound beams and is capable of describing the propagation of arbitrary sound beams in a wide variety of media. The algorithm used an operator-splitting algorithm to integrate the KZK equation. The 2D diffraction term was solved using a combined IBFD/CNFD method which we found to produce similar results to the ADI method but at lower computational cost. Results obtained from the algorithm were in excellent agreement with linear and nonlinear fields from a focused circular source; and the linear and nonlinear fields of unfocused rectangular sources. We note that the 3D code is computationally intensive but can be parallelized very effectively. Our simulations were carried out on an IBM pSeries 690 machine with 16 parallel processors; the tissue simulations shown in Figs. 10 and 11 each required a total of 9 CPU-hours and executed in about 0.6 wall-clock hours, which is close to the theoretical minimum of 0.56 wall-clock hours.

The code was used to simulate the field from a diagnostic ultrasound scanner. It was observed that the choice of absorption law had a strong effect on the prediction of the second-harmonic field. In comparing the spatial distribution of the fundamental and second-harmonic fields, we observed that the second-harmonic field was more sharply focused and that the second harmonic had much lower signal amplitude in the near field. These phenomena were present with both uniform and apodized sources. Both of these effects could be contributing factors to the improved imaging ability attributed to tissue harmonic imaging. The tighter focus should improve detail contrast, in particular by improving the lateral resolution. Clutter will be reduced both due to the narrower beam, which results in lower pressure levels off-axis, and due to the lower levels in the near-field, which results in less reverberation.

One effect not modeled with the 3D code at present is the effect of an inhomogeneous medium. The current model can be extended to account for small fluctuations in ambient properties. For variations in sound speed one may add a small fluctuation $c^{\prime}(x, y, z)$ that can vary in space to the background sound speed $c_{0}$. Within the ordering frame work of the KZK equation this introduces one extra term which essentially alters the phase to correct for the variation in sound speed. ${ }^{14}$ If the medium also has small variations in the density change to $\rho_{0}(x, y, z)$ then a new term $\nabla p \cdot \nabla \rho_{0} / \rho_{0}$ is introduced to the full wave equation. ${ }^{48}$ In the parabolic ap- proximation, to leading order, one finds that the density term reduces to

$$
\frac{1}{c_{0} \rho_{0}} \frac{\partial \rho_{0}}{\partial z} \frac{\partial p}{\partial t^{\prime}}-\frac{1}{\rho_{0}}\left(\frac{\partial \rho_{0}}{\partial x} \frac{\partial p}{\partial x}+\frac{\partial \rho_{0}}{\partial y} \frac{\partial p}{\partial y}\right) \text {. }
$$

Small scale variations in other parameters, i.e., nonlinearity, absorption, and dispersion, do not introduce extra terms into the second-order wave equation. For an inhomogeneous medium Eq. (1) can be rewritten in the following form:

$$
\begin{aligned}
\frac{\partial p}{\partial z}= & \frac{c_{0}}{2} \int_{-\infty}^{t^{\prime}}\left(\frac{\partial^{2} p}{\partial x^{2}}+\frac{\partial^{2} p}{\partial y^{2}}\right) d t^{\prime \prime}+\frac{\beta}{2 \rho_{0} c_{0}^{3}} \frac{\partial p^{2}}{\partial t^{\prime}}+\frac{\delta}{2 c_{0}^{3}} \frac{\partial^{2} p}{\partial t^{\prime 2}} \\
& +\sum_{v} \frac{c_{v}^{\prime}}{c_{0}^{2}} \int_{-\infty}^{t^{\prime}} \frac{\partial^{2} p}{\partial t^{\prime \prime 2}} e^{-\left(t^{\prime}-t^{\prime \prime}\right) / t_{v}} d t^{\prime \prime}+\frac{c^{\prime}}{c_{0}^{2}} \frac{\partial p}{\partial t} \\
& +\frac{1}{2 \rho_{0}} \frac{\partial \rho_{0}}{\partial z} p-\frac{c_{0}}{2 \rho_{0}} \int_{-\infty}^{t} \frac{\partial \rho_{0}}{\partial x} \frac{\partial p}{\partial x}+\frac{\partial \rho_{0}}{\partial y} \frac{\partial p}{\partial y} d t .
\end{aligned}
$$

The added terms can be incorporated in the current numerical scheme. The phase-speed term associated with sound speed could be included into the nonlinear term, as an amplitude-independent effect, and calculated using the Poisson solution. The transverse density fluctuation could be incorporated into the diffraction term using finite-differences to evaluate the first-order derivatives. The density fluctuation in the axial direction is the only term that requires a new equation to be included into the operator splitting paradigm:

$$
\frac{\partial p}{\partial z}=\frac{1}{2 \rho_{0}} \frac{\partial \rho_{0}}{\partial z} p .
$$

This is an ordinary first-order differential equation that can be approximated with a finite-difference solution

$$
p(x, y, z+\Delta z)=p(x, y, z) \frac{2 \rho_{0}(x, y, z+\Delta z)}{\rho_{0}(x, y, z+\Delta z)+\rho_{0}(x, y, z)} .
$$

This corresponds to the plane wave pressure transmission coefficient for the propagation between two media of different densities.

In conclusion the extension of the Texas code to 3D provides an effective tool for modeling arbitrary finiteamplitude beams. In its current form it can account for propagation of arbitrary beams in a thermoviscous and relaxing fluid. We speculate that it may be advantageous over frequency domain codes in problems that involve short pulsed wave forms, for example, shock wave therapy and the forward propagation problem in ultrasound imaging. The present formulation can be extended to account for propagation in inhomogeneous media.

\section{ACKNOWLEDGMENTS}

The authors would like to thank Yuan Jing for providing comments on this manuscript. Computing resources were provided by the Scientific Computing and Visualisation group at Boston University. Partial funding was provided by National Institutes of Health through Grant No. 
P01-DK53881 and by CenSSIS, the Center for Subsurface Sensing and Imaging Systems, under the Engineering Research Centers Program of the National Science Foundation (Award No. EEC-9986821).

${ }^{1}$ A. J. Coleman and J. E. Saunders, "A review of the physical properties and biological effects of the high amplitude acoustic fields used in extracorporeal lithotripsy," Ultrasonics 31, 75-89 (1993).

${ }^{2} \mathrm{~K}$. Hynynen, "Demonstration of enhanced temperature elevation due to nonlinear propagation of focused ultrasound in a dog's thigh in vivo," Ultrasound Med. Biol. 13, 85-91 (1987).

${ }^{3}$ S. Vaezy, R. Martin, H. Yaziji, P. Kaczkowski, G. Keilman, S. Carter, M. Caps, E. Y. Chi, M. Bailey, and L. A. Crum, "Hemostasis of Punctured Blood Vessels Using High-Intensity Focused Ultrasound," Ultrasound Med. Biol. 24, 903-910 (1998).

${ }^{4}$ E. L. Carstensen, W. K. Law, N. D. McKay, and T. G. Muir, "Demonstration of nonlinear acoustic effects at biomedical frequencies and intensities," Ultrasound Med. Biol. 6, 359-368 (1980).

${ }^{5}$ H. C. Starritt, M. A. Perkins, F. A. Duck, and V. F. Humphrey, "Evidence for ultrasonic finite-amplitude distortion in muscle using medical equipment," J. Acoust. Soc. Am. 77, 302-306 (1985).

${ }^{6}$ D. R. Bacon and E. L. Carstensen, "Increased heating by diagnostic ultrasound due to nonlinear propagation," J. Acoust. Soc. Am. 88, 26-34 (1990).

${ }^{7}$ B. Ward, A. C. Baker, and V. F. Humphrey, "Nonlinear propagation applied to the improvement of resolution diagnostic medical ultrasound," J. Acoust. Soc. Am. 101, 143-154 (1997).

${ }^{8}$ M. A. Averkiou, D. N. Roundhill, and J. E. Powers, "A new imaging technique based on the nonlinear properties of tissues," Proceedings of the IEEE Ultrasonics Symposium, IEEE Catalog No. 97CH36118, 1997, vol. 2, pp. $1561-1566$.

${ }^{9} \mathrm{~T}$. Christopher, "Finite amplitude distortion-based inhomogeneous pulse echo ultrasonic imaging," IEEE Trans. Ultrason. Ferroelectr. Freq. Control 44, 125-139 (1997)

${ }^{10}$ T. D. Mast, L. M. Hinkelman, M. J. Orr, V. W. Sparrow, and R. C. Waag, "Simulation of ultrasonic pulse propagation through the abdominal wall," J. Acoust. Soc. Am. 102, 1177-1190 (1997).

${ }^{11}$ E. A. Zabolotskaya and R. V. Khokhlov, "Quasi-plane waves in the nonlinear acoustics of confined beams," Sov. Phys. Acoust. 15, 35-40 (1969).

${ }^{12}$ V. P. Kuznetsov, "Equation of nonlinear acoustics," Sov. Phys. Acoust. 16, 467-470 (1970)

${ }^{13}$ S. I. Aanonsen, T. Barkve, J. Naze Tjøtta, and S. Tjøtta, "Distortion and harmonic generation in the nearfield of a finite amplitude sound beam," J. Acoust. Soc. Am. 75, 749-768 (1984).

${ }^{14}$ B. Lipkens, P. Blanc-Benon, L. Dallois, M. F. Hamilton, and D. T. Blackstock, "Propagation of finite amplitude sound through turbulence: Modeling with geometrical acoustics and the parabolic approximation," J. Acoust. Soc. Am. 111, 487-498 (2002).

${ }^{15}$ V. A. Khokhlova, M. V. Averianov, Ph. Blanc-Benon, and R. O. Cleveland, "Propagation of nonlinear acoustic signals through inhomogeneous moving media," Proceedings of the IEEE Ultrasonics Symposium, Montreal, Canada (in press).

${ }^{16}$ B. E. McDonald and W. A. Kuperman, "Time domain formulation for pulse propagation including nonlinear behavior at a caustic," J. Acoust. Soc. Am. 87, 1473-1481 (1990).

${ }^{17}$ Y. S. Lee and M. F. Hamilton, "Time-domain modeling of pulsed finiteamplitude sound beams," J. Acoust. Soc. Am. 97, 906-917 (1995).

${ }^{18}$ M. A. Averkiou and M. F. Hamilton, "Nonlinear distortion of short pulses radiated by plane and focused circular pistons," J. Acoust. Soc. Am. 102, 2539-2548 (1997).

${ }^{19}$ Y. A. Pishchalnikov, O. A. Sapozhnikov, and V. A. Khokhlova, "A Modification of the Spectral Description of Nonlinear Acoustic Waves with Discontinuities," Acoust. Phys. 42, 362-367 (1996).

${ }^{20}$ T. Christopher and K. J. Parker, "New approaches to nonlinear diffractive field propagation," J. Acoust. Soc. Am. 90, 488-499 (1991).

${ }^{21}$ J. Tavakkoli, D. Cathignol, R. Souchon, and O. A. Sapozhnikov, "Modeling of pulsed finite-amplitude focused sound beams in time domain," J. Acoust. Soc. Am. 104, 2061-2072 (1998).

${ }^{22}$ G. Wojcik, J. Mould, Jr, F. Lizzi, N. Abboud., M. Ostromogilsky, and D. Vaughan, "Nonlinear modeling of therapeutic ultrasound," Proceedings of IEEE Ultrasonics Symposium, IEEE Catalog No. 95CH35844, vol. 2, 1617-1621 (1995)
${ }^{23}$ I. M. Hallaj and R. O. Cleveland, "Single pulse simulation of the pressure and temperature fields in thermoviscus fluids using the FDTD method," Acoust. Res. Lett. Online http://ojps.aip.org/arlo published in J. Acoust. Soc. Am. 105, L7-12 (1999).

${ }^{24}$ C.-C. Shen and P.-C. Li, "Harmonic leakage and image quality degradation in tissue harmonic imaging," IEEE Trans. Ultrason. Ferroelectr. Freq. Control 48, 728-736 (2001).

${ }^{25}$ R. J. Zemp, J. Tavakkoli, and R. S. Cobbold, "Modeling of nonlinear ultrasound propagation in tissue from array transducers." J. Acoust. Soc. Am. 113, 139-152 (2003).

${ }^{26}$ A. C. Baker, A. M. Berg, A. Sahin, and J. N. Tjøtta, "The nonlinear pressure field of plane, rectangular apertures: Experimental and theoretical results," J. Acoust. Soc. Am. 97, 3510-3517 (1995).

${ }^{27}$ M. D. Cahill and A. C. Baker, "Numerical simulation of the acoustic field of a phased-array medical ultrasound scanner," J. Acoust. Soc. Am. 104, 1274-1283 (1998).

${ }^{28}$ A. Bouakaz, C. T. Lancee, and N. de Jong, "Harmonic ultrasonic field of medical phased arrays: Simulations and measurements," IEEE Trans. Ultrason. Ferroelectr. Freq. Control 50, 730-735 (2003).

${ }^{29}$ R. O. Cleveland, M. F. Hamilton, and D. T. Blackstock, "Time-domain modeling of finite-amplitude sound in relaxing fluids," J. Acoust. Soc. Am. 99, 3312-3318 (1996).

${ }^{30}$ W. F. Ames, Numerical Methods for Partial Differential Equations, 3rd ed. (Academic, New York, 1992).

${ }^{31}$ M. F. Hamilton, J. Naze Tjøtta, and S. Tjøtta, "Nonlinear effects in the far field of a directive sound source," J. Acoust. Soc. Am. 78, 202-216 (1985).

${ }^{32}$ T. S. Hart and M. F. Hamilton, "Nonlinear effects in focused beams," J. Acoust. Soc. Am. 84, 1488-1496 (1988).

${ }^{33}$ J. Naze Tjøtta, S Tjøtta, and E. H. Vefring, "Effects if focusing on the nonlinear interaction between two collinear finite amplitude sound beams," J. Acoust. Soc. Am. 89, 1017-1027 (1991).

${ }^{34} \mathrm{~B}$. Ystad and J. Berntsen, "Numerical solution of the KZK equation for focusing sources," Acta Acustica 3, 323-330 (1995).

${ }^{35}$ A. Taflove, Computational Electrodynamics: The Finite-difference Time Domain Method (Artech, Norwood, MA, 1995), pp. 158-160.

${ }^{36} \mathrm{~T}$. Christopher, "Source pre-biasing for improved second harmonic bubble-response imaging," IEEE Trans. Ultrason. Ferroelectr. Freq. Control 46, 556-563 (1999).

${ }^{37}$ Q.-H. Liu and J. Tao, "The perfectly matched layer for acoustic waves in absorptive media,” J. Acoust. Soc. Am. 102, 2072-2082 (1997).

${ }^{38}$ L. G. Ullate and J. L. San Emeterio, "A new algorithm to calculate the transient near-field of ultrasonic phased arrays," IEEE Trans. Ultrason. Ferroelectr. Freq. Control 39, 745-753 (1992).

${ }^{39}$ H. Becher, K. Tiemann, C. Pohl, N. C. Nanda, M. A. Averkiou, J. E. Powers, and B. Lüderitz, "Improvement in the endocardial border delineation using tissue harmonic imaging," Echocardiography 15, 511-517 (1998).

${ }^{40}$ R. J. Graham, W. Gallas, J. S. Gelman, L. Donelan, and R. E. Peverill, "An assessment of tissue harmonic versus fundamental imaging modes for echocardiographic measurements," J. Am. Soc. Echocardiogr. 14, 1191-1196 (2001)

${ }^{41}$ G. A. Whalley, G. D. Gamble, H. J. Walsh, S. P. Wright, S. Agewall, N. Sharpe, and R. N. Doughty, "Effect of tissue harmonic imaging and contrast upon between observer and test-retest reproducibility of left ventricular ejection fraction measurement in patients with heart failure," Eur. J. Heart Fail. 6, 85-93 (2004).

${ }^{42}$ K. Kubota, N. Hisa, Y. Ogawa, and S. Yoshida, "Evaluation of tissue harmonic imaging for breast tumors and axillary lymph nodes," Oncol. Rep. 9, 1335-1338 (2002).

${ }^{43}$ S. Tanaka, O. Oshikawa, T. Sasaki, T. Ioka, and H. Tsukuma "Evaluation of tissue harmonic imaging for diagnosis of focal liver lesions," Ultrasound Med. Biol. 26, 183-187 (2000).

${ }^{44}$ T. Schmidt, C. Hohl, P. Haage, M. Blaum, D. Honnef, C. Weibeta, G. Staatz, and R. W. Gunther, "Diagnostic accuracy of phase-inversion tissue harmonic imaging versus fundamental B-mode sonography in the evaluation of focal lesions of the kidney," Am J Roentgenol. 180, 1639-1647 (2003)

${ }^{45}$ J. E. Browne, A. J. Watson, N. M. Gibson, N. J. Dudley, and A. T. Elliott, "Objective measurements of image quality," Ultrasound Med. Biol. 30, 229-237 (2004)

${ }^{46}$ C. Pislaru, J. D. Hooge, S. V. Pislaru, E. Brandt, R. Cipic, C. E. 
Angermann, F. J. van de Werf, B. Bijnes, M. C. Herregods, and G. R. Sutherland, "Is there a change in myocardial nonlinearity during the cardiac cycle?" Ultrasound Med. Biol. 27, 389-398 (2001).

${ }^{47}$ T. L. Szabo, "Time domain wave equations for lossy media obeying a frequency power law," J. Acoust. Soc. Am. 96, 491-500 (1994).

${ }^{48}$ I. M. Hallaj, R. O. Cleveland, P. Barbone, S. G. Kargl, and R. A. Roy, "Amplitude degradation of time-reversed pulses in nonlinear absorbing thermoviscous fluids," Ultrasonics 38, 885-889 (2000). 